\title{
Protein phosphatase 1 suppresses androgen receptor ubiquitylation and degradation
}

\author{
Xiaming Liu ${ }^{1, *}$, Weiwei Han ${ }^{2, *}$, Sarah Gulla ${ }^{1}$, Nicholas I. Simon ${ }^{1}$, Yanfei Gao ${ }^{1}$, \\ Changmeng Cai ${ }^{1}$, Hongmei Yang ${ }^{3}$, Xiaoping Zhang ${ }^{2}$, Jihong Liư ${ }^{4}$, Steven P. Balk ${ }^{1}$, \\ Shaoyong Chen ${ }^{1}$ \\ ${ }^{1}$ Hematology-Oncology Division, Department of Medicine, Beth Israel Deaconess Medical Center and Harvard Medical School, \\ Boston, Massachusetts 02215, USA \\ ${ }^{2}$ Department of Urology, Union Hospital, Tongji Medical School, Huazhong University of Science and Technology, Wuhan \\ 430022, China \\ ${ }^{3}$ Department of Pathogen Biology, Tongji Medical School, Huazhong University of Science and Technology, Wuhan 430030, \\ China \\ ${ }^{4}$ Department of Urology, Tongji Hospital, Huazhong University of Science and Technology, Wuhan 430030, China \\ *These authors have contributed equally to this work \\ Correspondence to: Shaoyong Chen, e-mail: schen@bidmc.harvard.edu \\ Steven P. Balk, e-mail: sbalk@bidmc.harvard.edu
}

Keywords: androgen receptor, prostate cancer, protein phosphatase 1

Received: August 06, $2015 \quad$ Accepted: November 18, $2015 \quad$ Published: November 30, 2015

\section{ABSTRACT}

The phosphoprotein phosphatases are emerging as important androgen receptor $(A R)$ regulators in prostate cancer $(\mathrm{PCa})$. We reported previously that the protein phosphatase 1 catalytic subunit (PP1a) can enhance AR activity by dephosphorylating a site in the AR hinge region (Ser650) and thereby decrease AR nuclear export. In this study we show that PP1a increases the expression of wildtype as well as an S650A mutant AR, indicating that it is acting through one or more additional mechanisms. We next show that PP1a binds primarily to the AR ligand binding domain and decreases its ubiquitylation and degradation. Moreover, we find that the PP1a inhibitor tautomycin increases phosphorylation of AR ubiquitin ligases including SKP2 and MDM2 at sites that enhance their activity, providing a mechanism by which PP1a may suppress AR degradation. Significantly, the tautomycin mediated decrease in AR expression was most pronounced at low androgen levels or in the presence of the AR antagonist enzalutamide. Consistent with this finding, the sensitivity of LNCaP and C4-2 PCa cells to tautomycin, as assessed by PSA synthesis and proliferation, was enhanced at low androgen levels or by treatment with enzalutamide. Together these results indicate that PP1a may contribute to stabilizing AR protein after androgen deprivation therapies, and that targeting PP1a or the AR-PP1a interaction may be effective in castration-resistant prostate cancer (CRPC).

\section{INTRODUCTION}

The androgen receptor (AR) plays a central role in prostate cancer $(\mathrm{PCa})$ development and progression. Most patients initially respond to androgen deprivation therapy (ADT, surgical or medical castration), but the tumors inevitably recur despite castrate levels of androgen (castration-resistant prostate cancer, CRPC). AR expression is generally increased in CRPC, and one mechanism driving its activity is intratumoral synthesis of androgens from adrenal gland derived precursors or de novo from cholesterol [1-4]. This activity can be suppressed by drugs including abiraterone (which inhibits the enzyme CYP17A1 required for androgen synthesis) or by the direct AR antagonist enzalutamide, and both abiraterone and enzalutamide are now approved for treatment of CRPC [5, 6]. However, patients who respond to these agents generally relapse within a year, and AR 
appears to still be contributing to the growth of these relapsed tumors $[7,8]$. Therefore, there remains a critical need to identify further mechanisms contributing to AR activity.

AR mRNA is highly expressed in CRPC with the AR gene being amplified in many cases [9, 10], while epigenetic mechanisms can further enhance AR gene transcription [11, 12]. AR activity in CRPC also may be enhanced by multiple kinase signaling pathways that directly or indirectly increase AR protein stability or transcriptional activity at low androgen levels [13, 14]. Recent findings have also underscored the significance of protein phosphatases in regulating $\mathrm{AR}$ and in $\mathrm{PCa}$ development. Protein phosphatase 2A (PP2A) can bind to AR and suppress its activity by dephosphorylation of several sites $[15,16]$. The physiological relevance of PP2A in PCa development is supported by the identification of alterations in PP2A and its subunits in PCa model systems and clinical specimens during tumor progression [17-19]. In contrast to PP2A, we reported that the protein phosphatase 1 catalytic subunit (PP1 $\alpha$ ) can enhance AR activity by dephosphorylating a site in the hinge region, Ser650 [16]. Phosphorylation of this site was shown previously to enhance AR nuclear export [20], and we found that PP1 $\alpha$ inhibition decreased nuclear expression of wild-type AR, but not an S650A mutant AR [16].

In this study we show that PP1 $\alpha$ can also increase AR expression independently of S650 dephosphorylation. We find that PP $1 \alpha$ binds to the AR ligand binding domain and decreases AR ubiquitination and degradation, particularly at low androgen levels or in the presence of AR antagonists. Mechanistically, we show that $\mathrm{PP} 1 \alpha$ can dephosphorylate and inactivate ubiquitin ligases that target AR for ubiquitylation and degradation. Together these findings show that PP $1 \alpha$ can contribute to maintaining AR protein expression and activity in CRPC, and that targeting PP1 $\alpha$ or the AR-PP1 $\alpha$ interaction may be a novel therapeutic approach.

\section{RESULTS}

\section{PP1a can enhance AR activity independently of S650 phosphorylation site}

Phosphorylation of AR at S650 enhances its nuclear export and subsequent degradation [20]. We reported previously that $\mathrm{PP} 1 \alpha$ interacts with AR and dephosphorylates S650, thereby increasing nuclear AR in PCa cells [16]. In support of this conclusion, we showed that PP1 $\alpha$ inhibition with tautomycin decreased nuclear levels of the wild-type AR, but not an S650A mutant AR. However, in further studies we have found that $\mathrm{PP} 1 \alpha$ overexpression enhances the transcriptional activity of both the wild-type and S650A mutant AR, as assessed by co-transfection of AR, PP1 $\alpha$ and an AR regulated reporter gene into $\mathrm{HeLa}$ cells or $\mathrm{LNCaP} \mathrm{PCa}$ cells (Figure 1A). Moreover, the effects of PP1 $\alpha$ on the wild-type and S650A mutant AR were comparable, with the S650A having slightly more basal activity in the absence of cotransfected PP1 $\alpha$ (Figure 1B). To discount effects from endogenous AR in LNCaP cells, we also examined the role of $\mathrm{S} 650$ in a W741C mutant AR, for which bicalutamide acts as an agonist versus being an antagonist for wild-type AR [21]. Significantly, the bicalutamide stimulated activities of both the W741C and W741C/S650A double mutant ARs in LNCaP cells were similarly increased by PP $1 \alpha$ co-transfection (Figure 1C).

Together these results indicated that PP1 $\alpha$ may regulate AR activity by one or more additional S650 independent mechanisms. Consistent with our previous report [16], PP1 $\alpha$ overexpression in transient transfections increases AR protein (Figure 1D), and this increase could be prevented by treatment with the PP1 $\alpha$ inhibitor tautomycin (Supplementary Figure S1). While tautomycin at comparable concentrations can also inhibit PP2A, we showed previously that PP2A increases AR degradation and that the PP2Aspecific inhibitor fostriecin increases AR protein [16] (see also Supplementary Figure 2). PP1 $\alpha$ inhibition with tautomycin also decreased endogenous AR protein in both LNCaP and C4-2 cells, particularly in steroid-depleted medium without addition of androgen (dihydrotestosterone, DHT) (Figure 1E). Significantly, tautomycin similarly decreased expression of the transiently transfected S650A mutant AR in HeLa cells (Figure 1F), and the stably expressed S650A mutant AR in LNCaP cells (Figure 1G, and Supplementary Figure S3). In both cases this effect was most pronounced in steroid-depleted medium in the absence of exogenous DHT. These results show that one mechanism through which PP1 $\alpha$ can increase AR activity independently of S650 dephosphorylation is by increasing AR protein.

\section{Consensus PP1 $\alpha$-interacting KVFF motif in AR DNA binding domain is not essential for binding}

As PP1 $\alpha$ and AR can form a complex [16], we next focused on the identification of molecular determinants mediating the $\mathrm{PP} 1 \alpha-\mathrm{AR}$ interaction and their role in increasing AR protein. PP $1 \alpha$ has two consensus docking motifs shared by interacting partners, $\mathrm{R} / \mathrm{K}-\mathrm{x}(0,1)-\mathrm{V}-\mathrm{x}-\mathrm{F}$ and $\mathrm{F}-\mathrm{x}-\mathrm{x}-\mathrm{R} / \mathrm{K}-\mathrm{x}-\mathrm{R} / \mathrm{K}$, where $\mathrm{x}$ stands for any amino acid [22]. AR has one potential PP1 $\alpha$ binding motif, KVFF, locating in the DNA-binding domain (DBD) and in the vicinity of the zinc finger 1 that mediates DNA binding (Figure 2A). Interestingly, all class I steroid receptors (AR/GR/ $\mathrm{PR} / \mathrm{MR}$ ) except ER $\alpha$ possess this motif. Moreover, we found that PP1 $\alpha$ cotransfection could also enhance the 
transcriptional activity of GR and MR, but not ER $\alpha$, suggesting a possible role for this site in mediating an interaction with PP1 $\alpha$ (Supplementary Figure S4). Consistent with the essential function of this site for DNA binding, disruption of this motif by mutagenesis (KVFF to KAFA) resulted in complete transcriptional inactivation of AR (Figure 2B).

We then took a coimmunoprecipitation approach to assess for a role of this candidate motif in the AR-PP1 $\alpha$ interaction. As shown in figure 2C, wildtype and KAFA mutant AR pulled down comparable amounts of PP1 $\alpha$ in both $293 \mathrm{~T}$ and Cos 1 cell lines. As a complementary approach, we also used microcystinagarose beads to precipitate endogenous PP $1 \alpha$ and to assess its interaction with AR [16]. The results showed that in HeLa cells PP1 $\alpha$ associates both with the wildtype and the KAFA mutant AR (Figure 2D). To further substantiate the above findings, we next generated an AR-DBD fragment and its mutated counterpart (DBDKAFA) and assessed binding to PP1 $\alpha$. The results indicated that PP1 $\alpha$ may interact with the AR-DBD, but that this interaction was not mediated by the KVFF motif (Figure 2E). Together, these results indicated that the KVFF site plays minimal if any role in PP1 $1 \alpha-A R$ interaction.

\section{PP1 $\alpha$ interacts predominantly with the AR ligand-binding domain}

We next systematically assessed the association between PP1 $\alpha$ and a panel of constructs spanning the AR N-terminal domain (NTD) that bears the major transcriptional activation function, the central DNA-binding domain (DBD), and the C-terminal ligand-binding domain (LBD). Analysis in androgencontaining medium demonstrated that PP1 $\alpha$ could associate with the isolated NTD and LBD, with the inclusion of the DBD not causing an appreciable increase in binding (Figure 3A). Comparison with the inputs further indicates that $\mathrm{PP} 1 \alpha$ has higher affinity

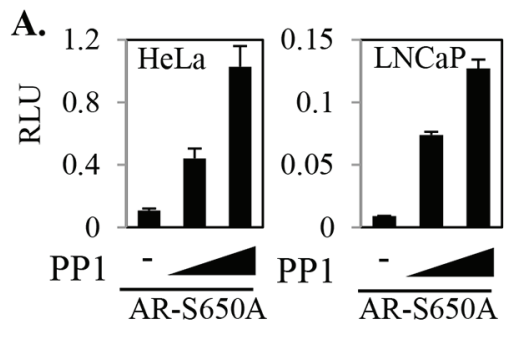

D.

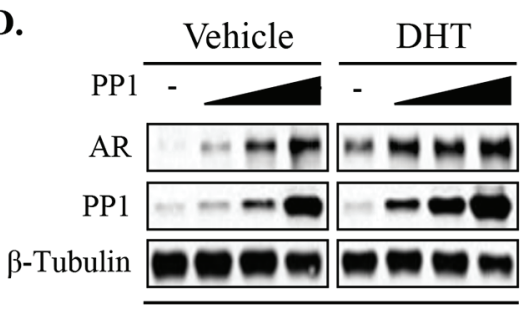

AR-WT

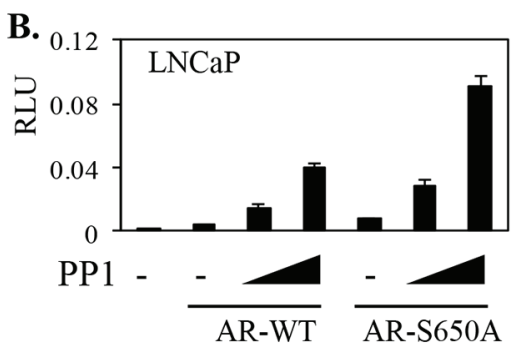

E.

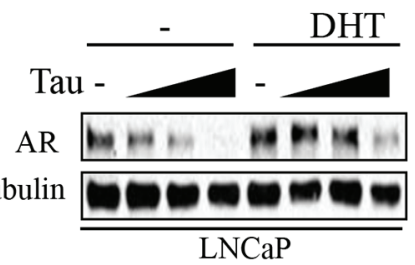

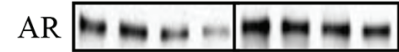

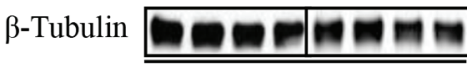

C4-2

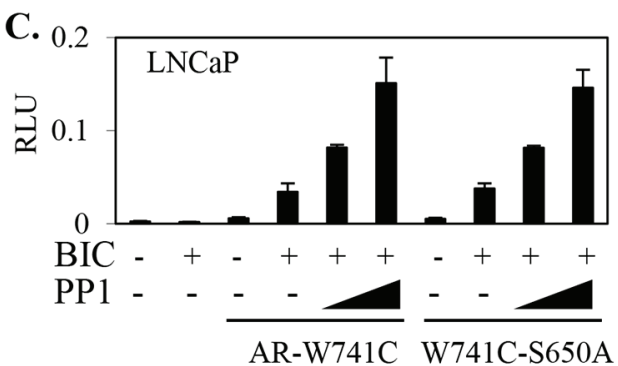

F.

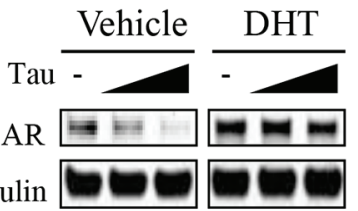

ARS650A

G.

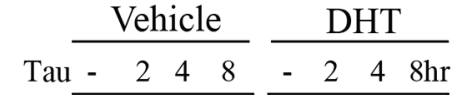

Flag-AR $-m-m=0$

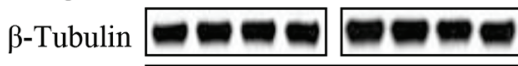

AR-S650A stable cell line

Figure 1: PP1 $\alpha$ can stabilize and activate AR independently of phospho-S650 dephosphorylation. A. HeLa and LNCaP cells were co-transfected with AR-S650A mutant and PP1 $\alpha$, together with ARE4-Luc reporter and CMV-Renilla (internal control). The cells were then incubated for overnight in androgen-depleted medium with DHT $(10 \mathrm{nM})$ for dual-Luc analyses. B. LNCaP cells were co-transfected with Renilla (internal control) and ARE4-Luc reporters, together without or with AR wild-type (WT) versus AR-S650A mutant and PP1 catalytic subunit (PP1 $\alpha$ ). The cells were treated for overnight with androgens (10 nM of DHT) for Dual-Luc analysis. C. LNCaP cells in androgen-depleted medium were co-transfected with Renilla and ARE4-Luc reporters, together with AR-W741C construct versus AR-W741C-S650A mutant and PP1 $\alpha$ as indicated. The cells were then incubated for overnight in androgen-depleted medium with bicalutamide (BIC, $10 \mu \mathrm{M})$ as indicated for Dual-Luc analysis. D. HeLa cells were transfected with Flag-PP1 $\alpha$ and HA-AR and then incubated for overnight in androgen-depleted medium without or with androgen (10 nM of DHT) as indicated for blotting. E. LNCaP and C4-2 cells in androgen-depleted medium were treated overnight with tautomycin (Tau, 100, 200, and $400 \mathrm{nM}$ ) and androgen (10 nM DHT for LNCaP and $1 \mathrm{nM}$ DHT for C4-2) as indicated and proteins were normalized for blotting. F, G. HeLa cells were transfected with AR S650A mutant (F) and a LNCaP line was generated to stably express Flag-tagged AR-S650A construct (G) Cells in androgen-depleted medium were treated for indicated time points with androgen ( $10 \mathrm{nM}$ of DHT) and tautomycin (Tau, $400 \mathrm{nM})$ and proteins were normalized for blotting. 
for the AR LBD than the NTD. We similarly observed preferential binding to the AR LBD when cells were cultured in steroid-depleted medium (Figure 3B). It should be noted while AR is primarily cytoplasmic in the absence of ligand and accumulates in the nucleus in response to androgen, $\mathrm{PP} 1 \alpha$ is highly expressed in the nucleus and cytoplasm (Supplementary Figure S5). Therefore, cellular localization is less likely to be a major factor dictating the association between PP1 $\alpha$ and these AR domains.

Notably, the PP1 $\alpha$-associated AR migrated on the gels as a major band with a series of distinct minor bands separated by $\sim 8 \mathrm{KDa}$, consistent with ubiquitylation. To confirm that these bands were ubiquitylated AR, we coexpressed histidine-tagged ubiquitin (his-Ub) together with PP1 $\alpha$ and AR, and then used Nickel(Ni)-NTA resin under denaturing conditions to pull-down ubiquitylated proteins. Immunoblotting for the HA-tagged AR then revealed higher molecular weight bands indicative of ubiquitylation associated primarily with constructs containing the LBD (Figure 3C). It should be noted that we also observed bands whose migration was consistent with the unmodified DBDLBD or LBD fragments (see also Supplementary Figure S6), which we presume reflect weak nonspecific binding to the resin compared to the ubiquitylated proteins that were highly enriched by the resin. We observed a similar pattern in the absence of transfected PP1 $\alpha$, consistent with the AR LBD being the primary target for ubiquitylation (Figure 3D).

\section{PP1 $\alpha$ stabilizes AR by decreasing AR LBD polyubiquitylation}

The above findings suggested that PP1 $\alpha$ may stabilize AR protein by decreasing ubiquitylation of the LBD. To examine this further, we assessed effects of PP1 $\alpha$ overexpression and proteasome inhibitor (MG132) treatment on ubiquitylation of the AR LBD. In the absence of transfected PP1 $\alpha$, treatment with MG132 increased both the levels of oligo- and poly-ubiquitylated AR LBD (Figure 4A). In contrast, these ubiquitylated $\mathrm{AR}$ species, and in particular the polyubiquitylated $\mathrm{AR}$, were not increased by MG132 in cells cotransfected with PP $1 \alpha$. Cotransfection of PP $1 \alpha$ similarly decreased the polyubiquitylation of full length AR (Figure 4B).

To determine whether this PP1 $\alpha$ mediated decrease in $\mathrm{AR}$ polyubiquitylation resulted in decreased $\mathrm{AR}$ degradation, we next used cycloheximide to block new protein synthesis and thereby assess AR protein stability. PP1 $\alpha$ cotransfection markedly decreased degradation of full length AR (Figure 4C). Moreover, consistent with PP1 $\alpha$ suppressing polyubiquitylation of the AR LBD, PP1 $\alpha$ cotransfection also markedly increased stability
A.

\section{AR
GR
PR
HR
ER}

Zinc Finger 1
Zinc Finger 2

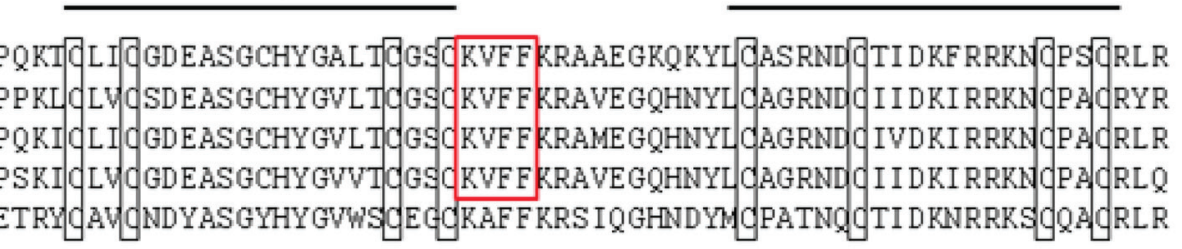

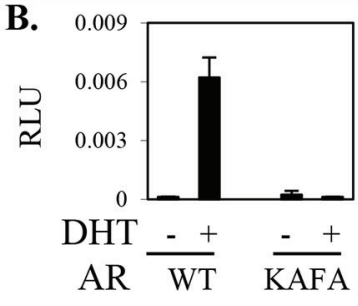

C.

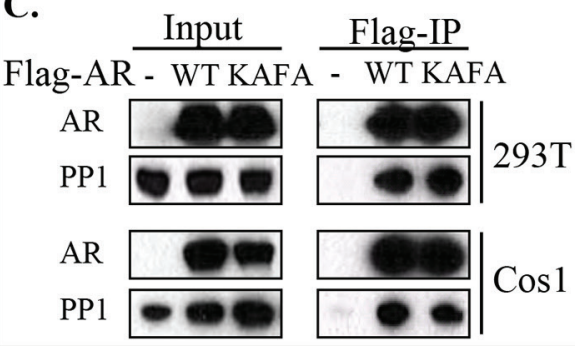

D.

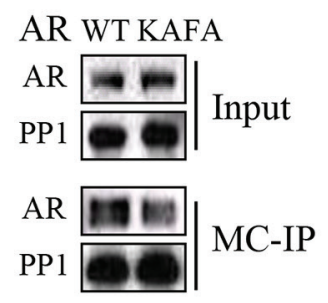

E.

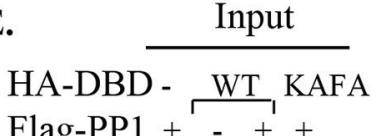

Flag-PPl +-++

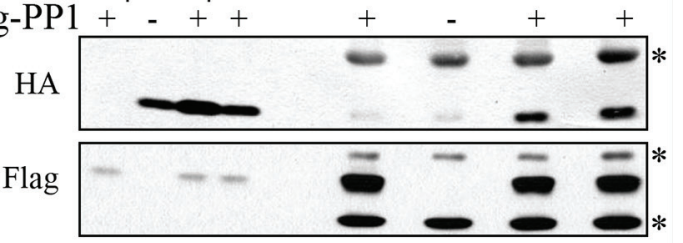

Figure 2: The potential PP1 $\alpha$-interacting KVFF motif of AR is not essential for AR-PP1 $\alpha$ interaction. A. A schematic drawing shows the linear amino acid sequence alignment of the zinc finger regions in the DNA binding domains of the class-I steroid receptors. Highlighted in the frame is the unique PP1 $\alpha$-interacting consensus motif $(\mathrm{KVxF})$ that exists in $\mathrm{AR}, \mathrm{GR}, \mathrm{PR}$, and MR, but not ER $\alpha$. Amino acid sequences are based on human androgen receptor (AR; GenBank M20132.1); human glucocorticoid receptor (GR $\alpha$; NM_001018077); human progesterone receptor (PR; M15716.1); human mineralocorticoid receptor (MR, NM_000901.3); and human estrogen receptor (ER $\alpha$; NM_000125.3). B. AR-deficient PCa cell line (PC3) was transfected with AR-mediated probasin-Luc and CMVRenilla reporters, together with AR wild-type (WT) versus KVFF mutant (KVFF to KAFA). Cells were then incubated for overnight in androgen-depleted medium with $10 \mathrm{nM}$ of DHT for Dual-Luc analysis. C. 293T and Cos1 cells were co-transfected with PP1 $\alpha$ and Flagtagged AR wild-type versus KVFF mutant (KAFA), with the empty Flag vector (-) as control. Co-immunoprecipitation (Co-IP) assay was carried out using anti-Flag-M2 beads. D. HeLa cells transfected with AR wild-type versus KAFA mutant were incubated for overnight in androgen-depleted medium with $10 \mathrm{nM}$ of DHT, followed by microcystin (MC)-IP and blotting. E. Flag-tagged PP1 $\alpha$ was co-transfected in 293 T cells with HA tagged AR-DBD wild-type (WT) versus DBD mutant (KAFA) constructs, followed by Co-IP using anti-Flag-M2 beads and blotting. Flag or HA empty vectors $(-)$ were used as controls, respectively. Non-specific IgG bands are marked $\left(^{*}\right)$. 
of the AR LBD, but not the AR NTD-DBD fragment (Figure 4C). Finally, PP1 $\alpha$ with an H248K mutation that inactivates its phosphatase activity was unable to increase AR protein, indicating that PP1 $\alpha$ stabilizes AR through dephosphorylation of one or more substrates (Figure 4D).

\section{PP1 $\alpha$ dephosphorylates ubiquitin ligases mediating AR degradation}

Several ubiquitin ligases, including MDM2 and SKP2, have been implicated as mediators of AR ubiquitylation and degradation [23-26]. MDM2 degradation can be enhanced by phosphorylation at multiple sites including S395, and previous reports indicate that PP1 $\alpha$ can decrease the degradation of MDM2 by dephosphorylating this site [27]. However, MDM2 activity can also be enhanced by growth factor stimulated phosphorylation of sites including S166 and S186 [28]. Effects of PP1 $\alpha$ on SKP2 have not been addressed previously. Therefore, we next assessed whether PP1 $\alpha$ may dephosphorylate and inactivate these ubiquitin ligases to attenuate AR ubiquitylation and degradation.

Consistent with previous reports, AR protein expression was decreased by cotransfection of MDM2
A.

Flag Sox9 $\frac{\text { Input }}{$\cline { 2 - 3 }}

$\frac{\text { Flag-IP }}{\operatorname{Sox} 9 \frac{\text { PP1 }}{}}$

HA-AR FL FL N N-D D D-L L

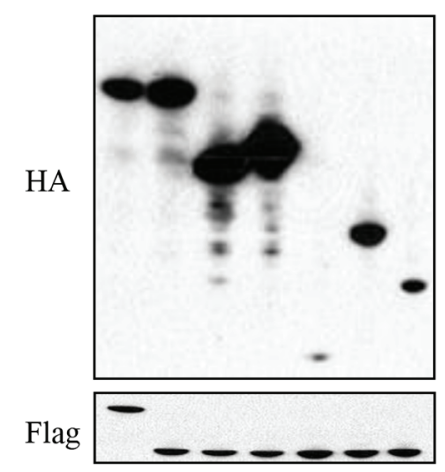

FL $\quad$ FL $\quad N \quad N-D \quad D \quad D-L \quad L$

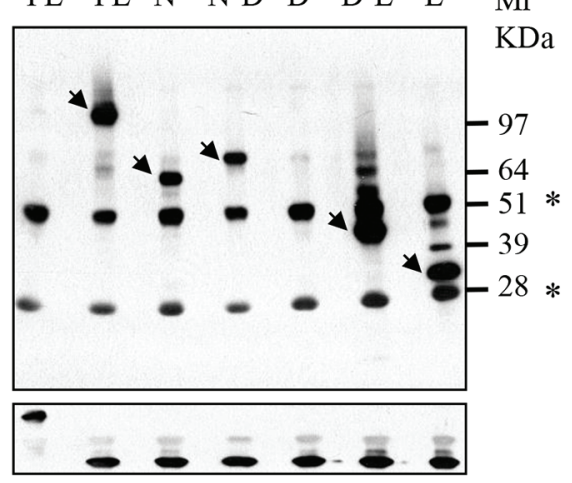

C.

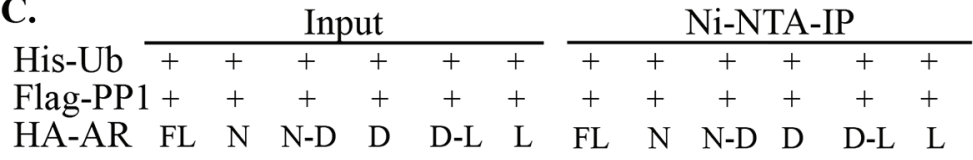

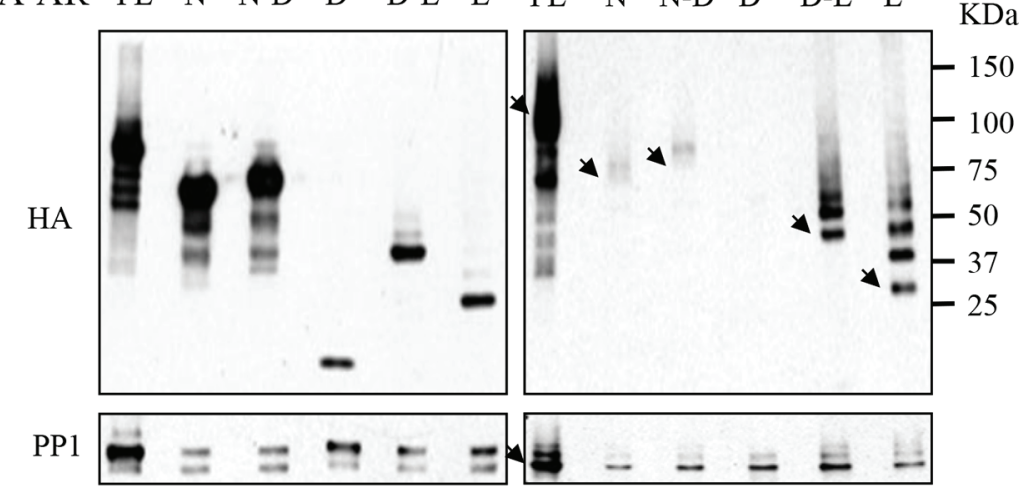

B.

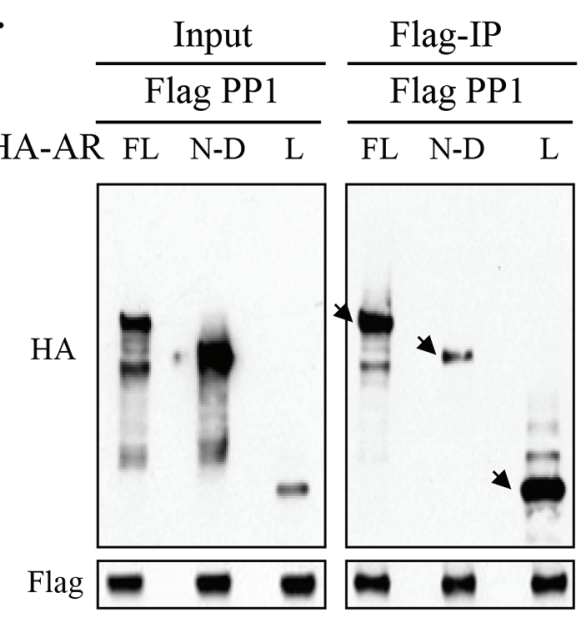

D.

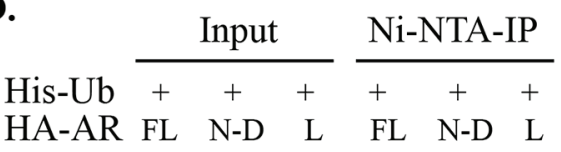

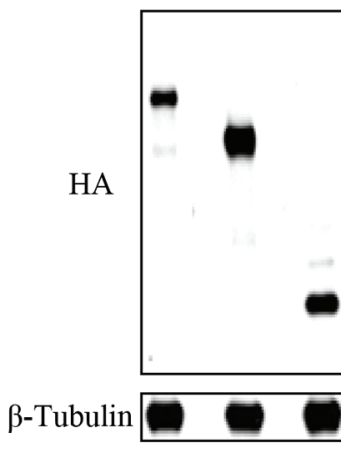

Figure 3: PP1 $\alpha$ engages predominantly with the AR ligand-binding domain (LBD) that is enriched in ubiquitination. A. $293 \mathrm{~T}$ cells in medium containing 5\% FBS were transfected with Flag-tagged PP1 $\alpha$ (Flag-PP1 $\alpha$ ) and HA-tagged AR full length (HA-AR FL) versus additional AR constructs (N: NTD; N-D: NTD-DBD; D: DBD; D-L: DBD-LBD; and L: LBD), followed by anti-Flag co-IP analysis for blotting. Arrows indicate native AR bands and non-specific IgG bands are marked (*). Flag-tagged Sox9, which weakly interacts with AR, was used as control. B. Similarly, 293T cells were transfected with Flag-PP1 $\alpha$ and HA-AR full length versus NTD-DBD and LBD constructs. After transfection, cells were then incubated for overnight in androgen-depleted medium for co-IP and blotting. C. 293T cells in androgendepleted medium were transfected with His-Ub, Flag-PP1 $\alpha$, and HA-AR constructs as indicated, followed by Ni(nickel)-NTA-IP under denatured condition and blotted. D. Similarly, 293T cells in androgen-depleted medium were transfected with His-Ub and indicated HA-AR constructs, followed by Ni-NTA-IP under denatured conditions. Arrowheads in the upper panels mark positions of the native AR constructs and an arrowhead on the lower panel of (C) marks the mono-ubiquitylated PP1 $\alpha$. Mr: Molecular weight marker in Kilodalton (KDa). 
or SKP2 (Figure 5A). Cotransfection of PP1 $\alpha$ resulted in a dramatic increase in the levels of transfected MDM2 and SKP2, which appears to be primarily due to increased transcription and translation from the expression vectors rather than decreased protein degradation. However, this may also in part reflect PP1 $\alpha$ mediated inactivation of MDM2 and SKP2 enzymatic activities, as this would suppress autoubiquitylation and degradation [29, 30]. In either case, despite the dramatic increases in MDM2 and SKP2, there were no increases in the ability of these proteins to suppress AR expression, indicating that their activity was markedly impaired by PP1 $\alpha$ (Figure 5A).

To determine whether endogenous PP1 $\alpha$ may regulate the phosphorylation and activity of these ubiquitin ligases, we treated $\mathrm{HeLa}$ and $\mathrm{LNCaP}$ cells with tautomycin and assessed for effects on endogenous MDM2 and SKP2. We did not observe clear increases in MDM2 or SKP2 in either cell line (Figure 5B). However, tautomycin increased MDM2 phosphorylation at S166, which has been reported to enhance MDM2 activity [28]. Similarly, tautomycin increased SKP2 phosphorylation at S72, a site that may be phosphorylated by AKT and lead to increased stability and activity $[31,32]$. Consistent with increased activity of MDM2 and SKP2, tautomycin treatment decreased levels of their respective substrates, p53 and p27 (Figure 5C).

Taken together these findings indicated that PP1 $\alpha$ inhibition may increase AR ubiquitylation and degradation by increasing the activity of one or more AR degrading ubiquitin ligases. However, it was not clear whether or how these effects on MDM2 of SKP2 were related to the PP1 $\alpha-\mathrm{AR}$ interaction. Therefore, we next asked whether AR could function as a scaffold to bridge PP1 $\alpha$ and MDM2 or SKP2. HeLa cells were transfected with Flag-tagged PP1 $\alpha$ and MDM2 or SKP2, with or without cotransfected AR. Anti-Flag immunoprecipitation then indicated that the PP1 $\alpha$-SKP2 association was substantially increased by cotransfected
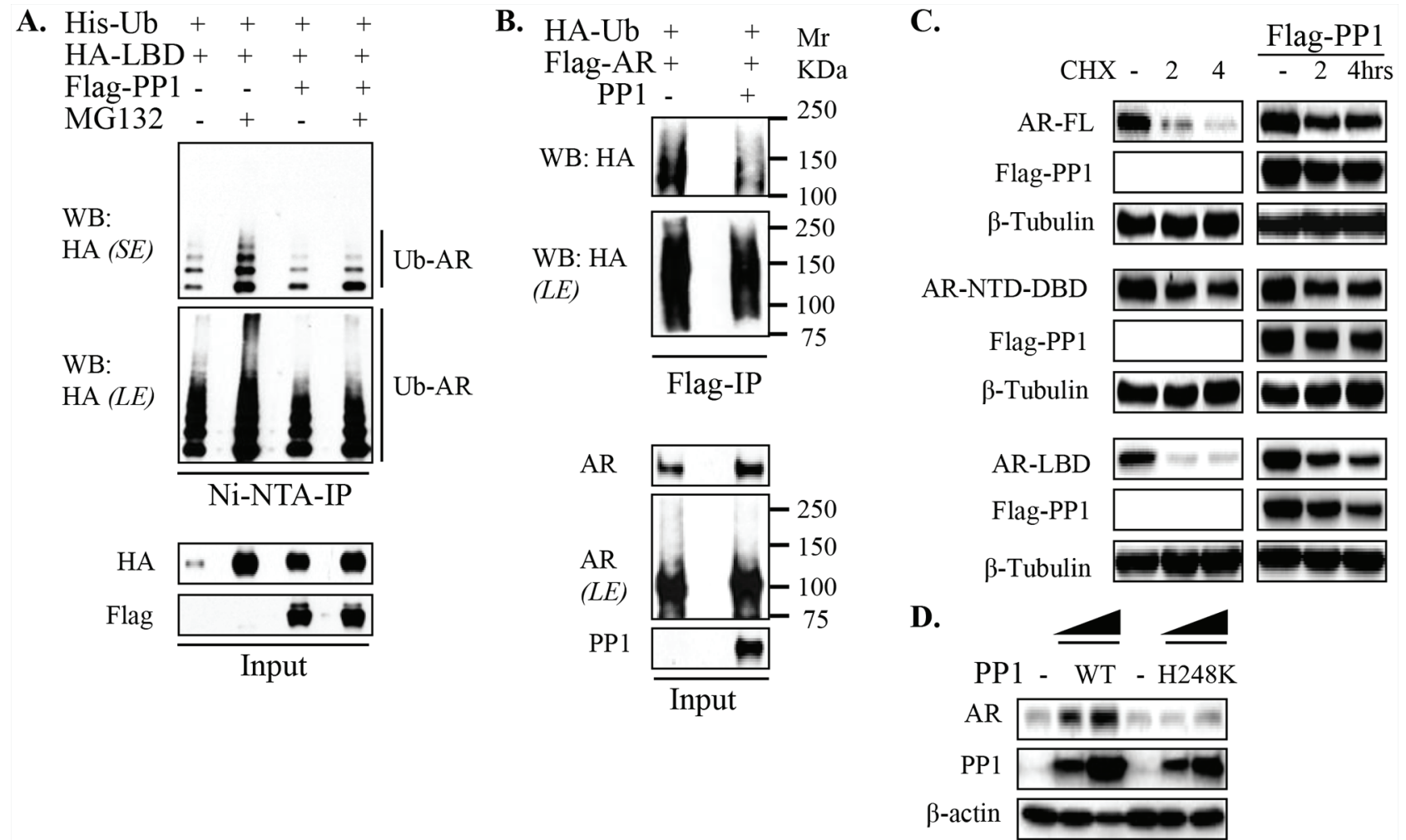

Figure 4: PP1 $\alpha$ stabilizes AR by attenuating LBD-directed AR ubiquitylation. A. 293T cells transfected with His-Ub, HAAR-LBD and Flag-PP1 $\alpha$ were incubated overnight in androgen-depleted medium without or with MG132 (10 $\mu \mathrm{M})$ as indicated, followed by Ni-NTA-IP under denatured conditions and blotting. SE: short exposure; $L E$ : long exposure. B. 293T cells were transfected with HAUb, Flag-AR-full-length together without or with PP1 $\alpha$. Cells were then incubated overnight in androgen-depleted medium, followed by anti-Flag-IP under denatured conditions. Mr: Molecular weight marker in Kilodalton (KDa). C. HeLa cells in androgen-depleted medium were co-transfected with HA-AR full-length (FL), NTD-DBD and LBD constructs, without or with Flag-PP1 $\alpha$ and cycloheximide as indicated. Total proteins were harvested and normalized for blotting. D. HeLa cells in androgen-depleted medium were co-transfected with AR and PP1 $\alpha$ wild-type versus its enzymatically inactive counterpart (H248K). Cells were then incubated in androgen-depleted medium for blotting. 
AR, consistent with a scaffold function (Figure 5D). AR cotransfection similarly increased the level of MDM2 that was coprecipitated with PP1 $\alpha$, but AR also increased input levels of MDM2, making interpretation of the result less clear (Figure 5D). Further studies to address this issue are now focused on identification of site directed mutations that can selectively impair the AR-PP1 $\alpha$ interaction.

\section{Androgen deprivation and $A R$ antagonists sensitize PCa cells to PP1 $\alpha$ inhibition}

As noted in Figure 1, effects of tautomycin on $\mathrm{AR}$ protein expression in $\mathrm{LNCaP}$ and $\mathrm{C} 4-2$ cells were most marked under conditions of androgen deprivation. Therefore, we compared the effects of tautomycin in C4-2 cells cultured in androgen depleted medium without or with added DHT. Consistent with its effects on AR protein under these conditions, tautomycin was more potent at reducing expression of PSA and proliferation under androgen depleted conditions (Figure 6A).

Significantly, effects of tautomycin on AR protein expression in $\mathrm{LNCaP}$ and $\mathrm{C} 4-2$ cells were also enhanced by treatment with the AR antagonist enzalutamide
(Figure 6B). Therefore, we examined the effects of tautomycin on AR activity in $\mathrm{LNCaP}$ and $\mathrm{C} 4-2$ cells without and with enzalutamide. In both cell lines, the inhibitory effects of tautomycin were enhanced by enzalutamide (Figure 6C). Collectively these findings indicate that agents targeting PP $1 \alpha$ may enhance the efficacy of androgen deprivation therapies and be effective in CRPC.

\section{DISCUSSION}

AR undergoes basal and ligand stimulated phosphorylation at multiple sites, and these modifications can modulate it stability, cellular localization and transcriptional activity. AR phosphorylation may also be regulated by protein phosphatases, including PP2A and PP1. PP2A can dephosphorylate multiple sites in the AR N-terminal domain, including S81, S94, S258, S308, and S424, that are normally increased in response to androgen and are associated with increased AR transcriptional activity [15]. Significantly, PP2A access to these sites may be negatively regulated by androgen binding, indicating that a physiological function of PP2A may be to suppress basal AR activation in the absence of androgen [33].
A.

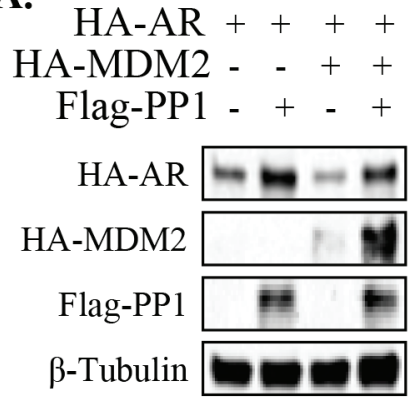

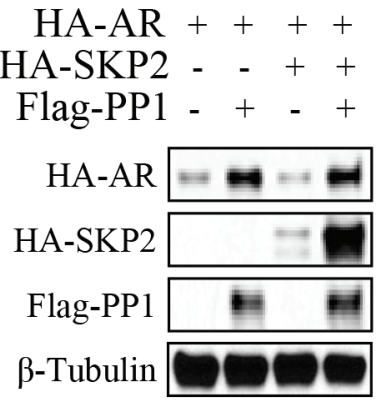

B.

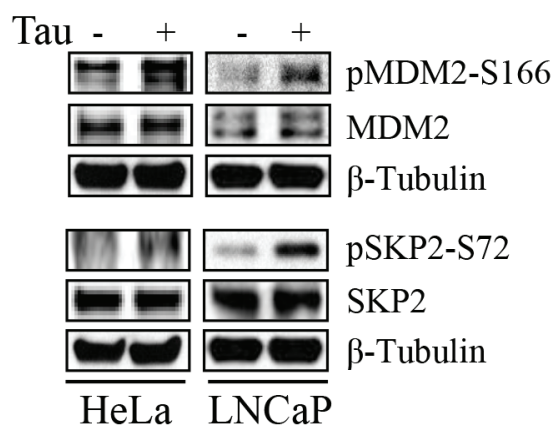

C.

D.

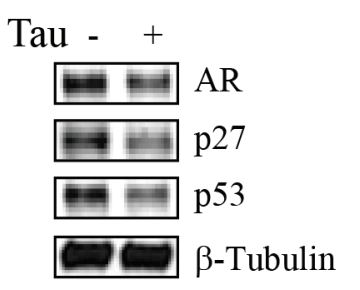

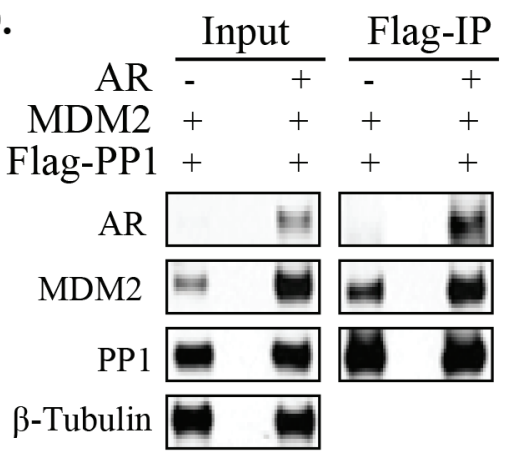

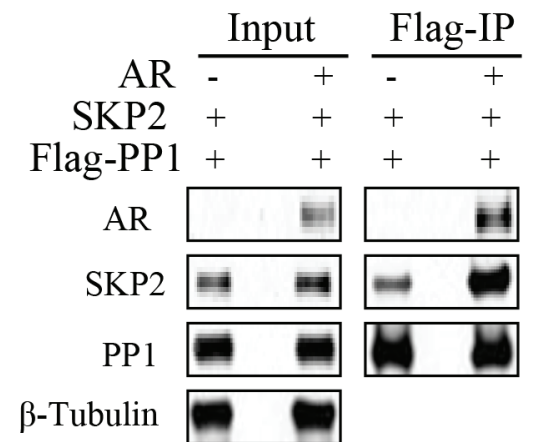

Figure 5: PP1 $\alpha$ can dephosphorylate AR degrading E3 ligases. A. HeLa cells were co-transfected with HA-AR, Flag-PP1 $\alpha$ and HA-tagged MDM2 or SKP2 as indicated. Cells were then incubated for overnight in androgen-depleted medium for blotting. B. HeLa and LNCaP cells in androgen-depleted medium were treated for $3 \mathrm{hrs}$ with $200 \mathrm{nM}$ of tautomycin, followed by protein normalization for blotting. C. LNCaP cells in androgen-depleted medium were treated for $3 \mathrm{hrs}$ with tautomycin as indicated. Total proteins were normalized for blotting. D. HeLa cells were transfected of Flag-PP1 and HA-MDM2 (left panel) or SKP2 (right panel), without or with AR as indicated. Cells were then incubated for overnight in androgen-depleted medium, followed by anti-Flag Co-IP and blotting. 
In contrast to PP2A, we had previously shown that PP $1 \alpha$ could bind to AR and dephosphorylate AR at S650 in the hinge region and thereby suppress AR nuclear export, providing a mechanism by which $\mathrm{PP} 1 \alpha$ can enhance AR activity [7]. In the current study we found that PP1 $\alpha$ could also suppress the polyubiquitylation of the AR LBD and its subsequent degradation, and that PP1 $\alpha$ inhibition with tautomycin could thereby decrease AR expression independently of S650 dephosphorylation. Mechanistically, we found that PP $1 \alpha$ inhibition with tautomycin increased the phosphorylation of MDM2 and SKP2 at sites that could enhance their activities, suggesting that PP1 $\alpha$ may suppress AR ubiquitylation by dephosphorylating these sites. Further results indicated that AR may mediate an interaction between PP1 $\alpha$ and these ubiquitin ligases, but further studies are needed to determine the relationship between the AR-PP $1 \alpha$ association and PP $1 \alpha$ mediated dephosphorylation of MDM2, SKP2, and potentially other ubiquitin ligases.

This study also found that PP $1 \alpha$ binding to AR is mediated primarily by the AR LBD. Although the precise site on the LBD remains to be determined, it does not appear to be the coactivator binding site as binding is not ligand dependent. We also cannot rule out that binding is indirect and mediated by a not yet identified PP1 regulatory protein. An attractive hypothesis is that $\mathrm{PP} 1 \alpha$ associated with the AR LBD directly protects AR from ubiquitylation by locally inactivating ubiquitin ligases. Alternatively, PP1 $\alpha$ may more globally suppress a subset of ubiquitin ligases, with the AR associated PP $1 \alpha$ having distinct functions including the dephosphorylation of S650. Further studies are underway to test these hypotheses.

In any case, these findings indicate that therapies targeting PP1 $\alpha$, or possibly the AR-PP1 $\alpha$ interaction, may have efficacy in CRPC. Significantly, the decrease in AR protein in response to tautomycin was most dramatic under androgen depleted conditions and in cells treated with the AR antagonist enzalutamide. Therefore, PP1 $\alpha$ activity may play a particularly important role in maintaining AR expression in CRPC and in resistance to enzalutamide.
A.

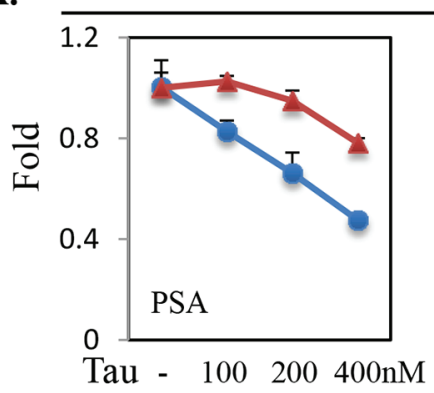

C4-2

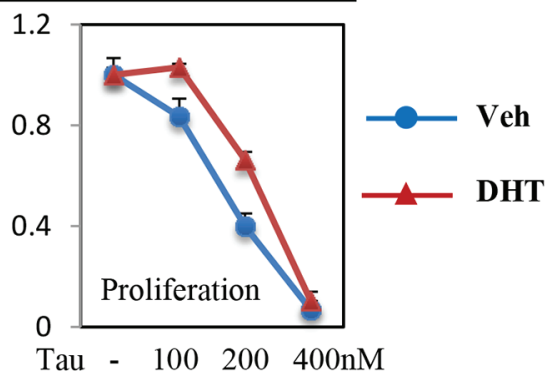

B.

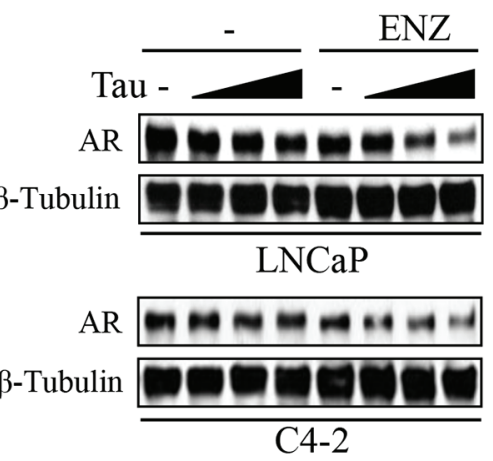

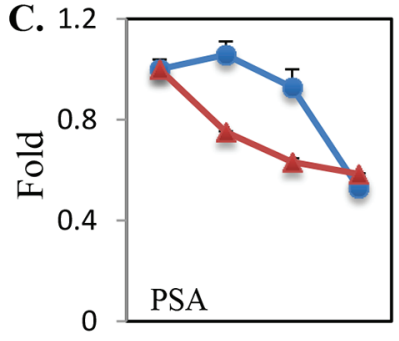

Tau - $100200 \quad 400 \mathrm{nM}$

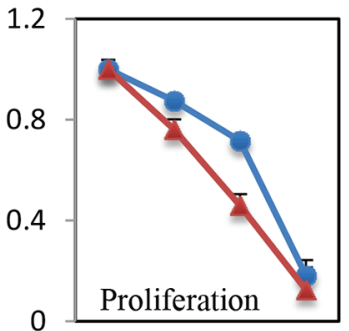

- $100200400 \mathrm{nM}$

$\mathrm{LNCaP}$
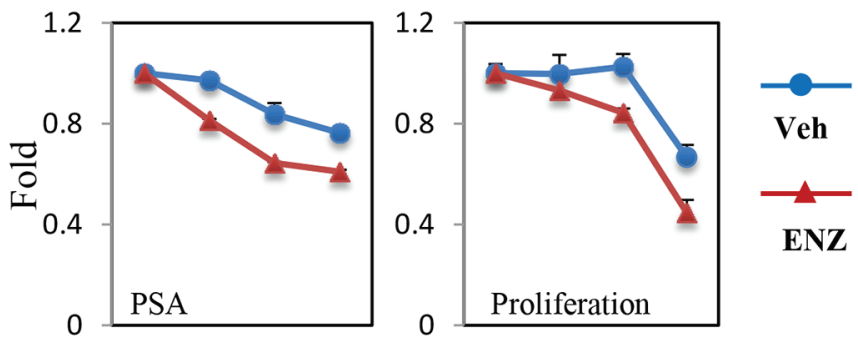

Tau - $100200400 \mathrm{nM} \quad-100200 \quad 400 \mathrm{nM}$

$\mathrm{C} 4-2$

Figure 6: Androgen ablation increases PP1 $\alpha$-dependence for AR protein expression and transactivation in PCa cells. A. C4-2 cells in androgen-depleted medium were treated with tautomycin and androgen (1nM DHT) for qRT-PCR analysis of PSA expression ( $24 \mathrm{hr}$ treatment) or for cell proliferation (3-day treatment), with the results being normalized to the no tautomycin levels in the absence and presence of DHT. Veh: vehicle; DHT: $1 \mathrm{nM}$ of DHT. B, C. LNCaP and C4-2 cells in androgen-containing medium (10\% FBS) were treated with enzalutamide (ENZ, $10 \mu \mathrm{M})$ and tautomycin $(100,200$, and $400 \mathrm{nM})$ as indicated. Total proteins (for $24 \mathrm{hr}$ treatment) were harvested and normalized for blotting (B) and total RNA (for $24 \mathrm{hr}$ treatment) was isolated for qRT-PCR analysis of PSA expression (C). The cell proliferation analysis was carried out after 3-day treatment $(C)$. The gene expression and cell growth results were normalized to no tautomycin in the absence and presence of enzalutamide (C). Veh: vehicle; Enz: $10 \mu \mathrm{M}$ of enzalutamide. 


\section{MATERIALS AND METHODS}

\section{Materials}

Anti-Flag M2 affinity gel was from Sigma-Aldrich, tautomycin and fostriecin were from Calbiochem and Enzo Life Science, Ni-NTA agarose was from Qiagen, and microcystin-agarose was from EMD Millipore. AntiAR, anti-PP1, anti-HA, anti-p27, and anti-p53 antibodies were from Cell Signaling, anti- $\beta$-Actin was from Abcam, anti- $\beta$-tubulin was from EMD Millipore, and anti-flag-M2 was from Sigma-Aldrich. The anti-SKP2 (Santa Cruz) and anti-p-SKP2-S72 (Cell Signaling) antibodies were kindly provided by Dr. Inuzuka Hiroyuki, and the anti-MDM2 and anti-p-MDM2-S166 (Santa Cruz) antibodies were kindly provided by Dr. Ming Chen (BIDMC, Harvard Medical School, Boston, USA). FBS and CDS (charcoaldextran stripped serum) were from Hyclone.

\section{Immunoprecipitations (IPs)}

For coimmunoprecipitation (co-IP), cells were harvested in triton lysis buffer (TLB) containing protease inhibitors. The lysates were incubated on ice for $20 \mathrm{~min}$ and centrifuged for $5 \mathrm{~min}$ at maximum speed. $20 \mu \mathrm{l}$ of the supernatant was saved as input and the rest was incubated with anti-Flag beads, followed by incubation at $4^{\circ} \mathrm{C}$ on a rotator for $2 \mathrm{hr}$. Then the input and washed (4x) IP beads were boiled in $60 \mu \mathrm{l}$ of Laemmli buffer containing $5 \% \beta$-mercaptoethanol. For immunoprecipitation (IP) under denatured conditions, cells were harvested by boiling in $2 \%$ SDS followed by $20 x$ dilution the TLB. The test then proceeded as the above regular IP. For the ubiquitylation and Ni(nickel)-NTA-IP assay, the cells were washed in PBS and an aliquot was lysed in RIPA buffer as input. The rest was lysed in buffer A (6M guanidine $\mathrm{HCl}$, 0.1 M Na2HPO4/NaH2PO4, $10 \mathrm{mM}$ imidazole, $\mathrm{pH} 8.0$ ), followed by sonication and centrifugation. The supernatants were incubated with Ni-NTA beads at room temperature for $3 \mathrm{hrs}$, followed by wash $2 \mathrm{x}$ with buffer $\mathrm{A}$, $2 \mathrm{x}$ with buffer A/buffer TI (1:3), and $1 \mathrm{x}$ with buffer TI (25 $\mathrm{mM}$ Tris-Cl, $20 \mathrm{mM}$ imidazole, $\mathrm{pH}$ 6.8). Then both input and the IP yields were boiled in $60 \mu$ of Laemmli buffer containing 5\% $\beta$-mercaptoethanol. Microcystin-IP was as previously described [16].

\section{Gene expression analysis}

RNA isolation was carried out using the TriZOL reagent and the qRT-PCR analysis on gene expression was performed with the TaqMan One-Step RT-PCR Master Mix Reagents (Applied Biosystems). The TaqMan primer and probe sets for gene expression assay: PSA: Forward, 5'- GATGAAACAGGCTGTGCCG-3'; Reverse, 5'- CCTCACAGCTACCCACTGCA-3'; Probe, 5'- FAMCAGGAACAAAAGCGTGATCTTGCTGGG-3'. The TaqMan primer-probe set for GAPDH transcripts (internal control) was purchased as inventoried mix from Applied Biosystems.

\section{Cell culture, cellular fractionation, transient transfection and reporter gene assays}

LNCaP cells were grown in the RPMI-1640 medium and $\mathrm{C} 4-2$ and $\mathrm{VCaP}$ cells in DMEM medium containing $10 \%$ FBS. PC3, 293 T, HeLa, and Cos1 cells were grown in DMEM medium with 5\% FBS. For androgen-starved conditions, cells were grown in medium containing 5\% CDS. Cellular fractionation assay were carried out with the NE-PER kit (Pierce), following the manufacture's direction. For transfection, cells were grown in normal growth medium to $\sim 80 \%$ confluence and plasmid DNA was transfected with LipofectAMINE 2000. Empty relevant vectors (such as pCDNA3.1, HA vector, and Flag vector) were supplemented to equalize the total amount of plasmids in the transfections. After overnight transfection, the cultures were refreshed with indicated medium for treatments. The pCMV-pRL-Renilla and androgenresponsive reporter plasmids have been described previously [16]. Additional steroids, steroids receptors and reporters were from the Balk lab. Luciferase activities were measured with the Dual-Luc assay kit (Promega). The ratios between Firefly and Renilla luciferase activities are shown as relative light units (RLU), and the results reflect the mean and standard deviation from triplicate samples.

\section{Cell proliferation assay}

Cells were cultured in 96-well plates for 2 days and then treated for 3 days as indicated. The cell proliferation analysis was performed with the CellTiter-Glo assay kit (Promega), following the manufacture's manual.

\section{Western blot data processing}

The Western blotting was developed based on $\mathrm{X}$-Ray film (Research Products International) and the western lightning Plus-ECL reagent (PerkinElmer). The images were acquired using the CanoScan LiDE 210 scanner and processed using the Adobe Photoshop 7.0 software. Relevant images were quantified by using the ImageJ software. All gels shown are representative of results from at least three experiments.

\section{Plasmids and DNA mutagenesis}

The AR constructs were generated based on the pcDNA3.1(+)-Kozak-HA-HA vector and cover the following regions: HA-HA-AR full-length (aa 1-919); HA-HA-AR-NTD (aa 1-539); HA-HA-AR-NTD-DBD (aa 1-628); HA-HA-AR-DBD (aa 538-628); and HAHA-AR-DBD-LBD (aa 538-919). The HA-AR-LBD (aa 662-919) vector was from our Lab. The untagged PP1 $\alpha$ plasmid was from Origene and then cloned into the 
3x-Flag-CMV-10 vector (Sigma-Aldrich) to generate the Flag-PP1 $\alpha$ expression plasmid. The his-Ub, Flag-Ub, FlagSox9, HA-MDM2 and HA-SKP2 expression plasmids were kindly provided by Drs. Pengda Liu, Wenjian Gan, Xin Yuan, and Inuzuka Hiroyuki (BIDMC), respectively. DNA mutagenesis on AR and PP1 $\alpha$ was performed with the Quick-change site-directed mutagenesis Kit (Stratagene). Details for plasmids and mutants generation are available upon request.

\section{ACKNOWLEDGMENTS}

We thank Drs. Pengda Liu, Wenjian Gan, Inuzuka Hiroyuki, Ming Chen, Greg Finn, and Xin Yuan (BIDMC, Harvard Medical School, Boston, USA) for reagents and/ or technical assistance.

\section{CONFLICTS OF INTEREST}

The authors declare no conflict of interest.

\section{GRANT SUPPORT}

This work was supported by grants from NIH (K99/R00 grant CA135592) to SYC, NIH P01 grant (CA163227) and SPORE in Prostate Cancer P50 grant (CA090381) to SPB, and a DOD Idea Development Award (W81XWH-13-1-0266) to SPB. XML is partly supported by a grant (No: 2013CFB095, HuBei Natural Science Foundation, China). WWH is supported by grants to XPZ (Grant No. 30872924, 81072095 \& 81372760 from the National Natural Science Foundation of China; Grant No. NCET-08-0223 from the Program for New Century Excellent Talents in University from the Department of Education of China; and Grant No. 2012AA021101 from the National High Technology Research and Development Program of China (863 Program) and grants to HMY (Grant No. 31070142 \& 81272560 from the National Natural Science Foundation of China).

\section{Author Contributions}

X.M.L. and W.W.H. performed all experiments; N.S. and S.G. participated in Co-IP tests; Y.F.G. participated in blotting and experimental design; C.M.C., H.M.Y., X.P.Z. and J.H.L. participated in experimental design; S.P.B. and S.Y.C. were engaged in all aspects and wrote the paper.

\section{REFERENCES}

1. Titus MA, Schell MJ, Lih FB, Tomer KB and Mohler JL. Testosterone and dihydrotestosterone tissue levels in recurrent prostate cancer. Clin Cancer Res. 2005; 11:4653-4657.

2. Stanbrough M, Bubley GJ, Ross K, Golub TR, Rubin MA, Penning TM, Febbo PG and Balk SP. Increased expression of genes converting adrenal androgens to testosterone in androgen-independent prostate cancer. Cancer Res. 2006; 66:2815-2825.

3. Montgomery RB, Mostaghel EA, Vessella R, Hess DL, Kalhorn TF, Higano CS, True LD and Nelson PS. Maintenance of intratumoral androgens in metastatic prostate cancer: a mechanism for castration-resistant tumor growth. Cancer Res. 2008; 68:4447-4454.

4. Locke JA, Guns ES, Lubik AA, Adomat HH, Hendy SC, Wood CA, Ettinger SL, Gleave ME and Nelson CC. Androgen levels increase by intratumoral de novo steroidogenesis during progression of castration-resistant prostate cancer. Cancer Res. 2008; 68:6407-6415.

5. Ryan CJ, Smith MR, de Bono JS, Molina A, Logothetis CJ, de Souza P, Fizazi K, Mainwaring P, Piulats JM, Ng S, Carles J, Mulders PF, Basch E, Small EJ, Saad F, Schrijvers D, et al. Abiraterone in metastatic prostate cancer without previous chemotherapy. N Engl J Med. 2013; 368:138-148.

6. Beer TM, Armstrong AJ, Rathkopf DE, Loriot Y, Sternberg $\mathrm{CN}$, Higano CS, Iversen P, Bhattacharya S, Carles J, Chowdhury S, Davis ID, de Bono JS, Evans CP, Fizazi K, Joshua AM, Kim CS, et al. Enzalutamide in metastatic prostate cancer before chemotherapy. N Engl J Med. 2014; 371:424-433.

7. Chen EJ, Sowalsky AG, Gao S, Cai C, Voznesensky O, Schaefer R, Loda M, True LD, Ye H, Troncoso P, Lis RL, Kantoff PW, Montgomery RB, Nelson PS, Bubley GJ, Balk SP, et al. Abiraterone treatment in castration-resistant prostate cancer selects for progesterone responsive mutant androgen receptors. Clin Cancer Res. 2015; 21:1273-1280.

8. Robinson D, Van Allen EM, Wu YM, Schultz N, Lonigro RJ, Mosquera JM, Montgomery B, Taplin ME, Pritchard CC, Attard G, Beltran H, Abida W, Bradley RK, Vinson $\mathrm{J}$, Cao X, Vats P, et al. Integrative clinical genomics of advanced prostate cancer. Cell. 2015; 161:1215-1228.

9. Taplin ME, Bubley GJ, Shuster TD, Frantz ME, Spooner AE, Ogata GK, Keer HN and Balk SP. Mutation of the androgen-receptor gene in metastatic androgen-independent prostate cancer. N Engl J Med. 1995; 332:1393-1398.

10. Visakorpi T, Hyytinen E, Koivisto P, Tanner M, Keinanen $\mathrm{R}$, Palmberg C, Palotie A, Tammela T, Isola $\mathrm{J}$ and Kallioniemi OP. In vivo amplification of the androgen receptor gene and progression of human prostate cancer. Nat Genet. 1995; 9:401-406.

11. Cai C, He HH, Chen S, Coleman I, Wang H, Fang Z, Nelson PS, Liu XS, Brown M and Balk SP. Androgen receptor gene expression in prostate cancer is directly suppressed by the androgen receptor through recruitment of lysine-specific demethylase 1. Cancer Cell. 2011; 20:457-471.

12. Sharma A, Yeow WS, Ertel A, Coleman I, Clegg N, Thangavel C, Morrissey C, Zhang X, Comstock CE, Witkiewicz AK, Gomella L, Knudsen ES, Nelson PS and Knudsen KE. The retinoblastoma tumor suppressor controls 
androgen signaling and human prostate cancer progression. J Clin Invest. 2010; 120:4478-4492.

13. Yuan X, Cai C, Chen S, Chen S, Yu Z and Balk SP. Androgen receptor functions in castration-resistant prostate cancer and mechanisms of resistance to new agents targeting the androgen axis. Oncogene. 2014; 33:2815-2825.

14. Koryakina Y, Ta HQ and Gioeli D. Androgen receptor phosphorylation: biological context and functional consequences. Endocr Relat Cancer. 2014; 21:T131-145.

15. Yang CS, Vitto MJ, Busby SA, Garcia BA, Kesler CT, Gioeli D, Shabanowitz J, Hunt DF, Rundell K, Brautigan DL and Paschal BM. Simian virus 40 small $t$ antigen mediates conformation-dependent transfer of protein phosphatase 2A onto the androgen receptor. Mol Cell Biol. 2005; 25:1298-1308.

16. Chen S, Kesler CT, Paschal BM and Balk SP. Androgen receptor phosphorylation and activity are regulated by an association with protein phosphatase 1. J Biol Chem. 2009; 284:25576-25584.

17. Bluemn EG, Spencer ES, Mecham B, Gordon RR, Coleman I, Lewinshtein D, Mostaghel E, Zhang X, Annis J, Grandori C, Porter C and Nelson PS. PPP2R2C loss promotes castration-resistance and is associated with increased prostate cancer-specific mortality. Mol Cancer Res. 2013; 11:568-578.

18. Cheng Y, Liu W, Kim ST, Sun J, Lu L, Zheng SL, Isaacs $\mathrm{WB}$ and $\mathrm{Xu}$ J. Evaluation of PPP2R2A as a prostate cancer susceptibility gene: a comprehensive germline and somatic study. Cancer Genet. 2011; 204:375-381.

19. Pandey P, Seshacharyulu P, Das S, Rachagani S, Ponnusamy MP, Yan Y, Johansson SL, Datta K, Fong Lin $\mathrm{M}$ and Batra SK. Impaired expression of protein phosphatase 2A subunits enhances metastatic potential of human prostate cancer cells through activation of AKT pathway. Br J Cancer. 2013; 108:2590-2600.

20. Gioeli D, Black BE, Gordon V, Spencer A, Kesler CT, Eblen ST, Paschal BM and Weber MJ. Stress kinase signaling regulates androgen receptor phosphorylation, transcription, and localization. Mol Endocrinol. 2006; 20:503-515.

21. Chen S, Gulla S, Cai C and Balk SP. Androgen receptor serine 81 phosphorylation mediates chromatin binding and transcriptional activation. J Biol Chem. 2012; 287:8571-8583.

22. Garcia A, Cayla X, Caudron B, Deveaud E, Roncal F and Rebollo A. New insights in protein phosphorylation: a signature for protein phosphatase 1 interacting proteins. C R Biol. 2004; 327:93-97.
23. Lin $\mathrm{HK}$, Wang $\mathrm{L}, \mathrm{Hu} \mathrm{YC}$, Altuwaijri S and Chang C. Phosphorylation-dependent ubiquitylation and degradation of androgen receptor by Akt require Mdm2 E3 ligase. EMBO J. 2002; 21:4037-4048.

24. Gaughan L, Logan IR, Neal DE and Robson CN. Regulation of androgen receptor and histone deacetylase 1 by Mdm2mediated ubiquitylation. Nucleic Acids Res. 2005; 33:13-26.

25. Chymkowitch P, Le May N, Charneau P, Compe E and Egly JM. The phosphorylation of the androgen receptor by TFIIH directs the ubiquitin/proteasome process. EMBO J. 2011; 30:468-479.

26. Li B, Lu W, Yang Q, Yu X, Matusik RJ and Chen Z. Skp2 regulates androgen receptor through ubiquitin-mediated degradation independent of Akt/mTOR pathways in prostate cancer. Prostate. 2014; 74:421-432.

27. Lee SJ, Lim CJ, Min JK, Lee JK, Kim YM, Lee JY, Won $\mathrm{MH}$ and Kwon YG. Protein phosphatase 1 nuclear targeting subunit is a hypoxia inducible gene: its role in post-translational modification of p53 and MDM2. Cell Death Differ. 2007; 14:1106-1116.

28. Zhou BP, Liao Y, Xia W, Zou Y, Spohn B and Hung MC. HER-2/neu induces p53 ubiquitination via Akt-mediated MDM2 phosphorylation. Nat Cell Biol. 2001; 3:973-982.

29. Fang S, Jensen JP, Ludwig RL, Vousden KH and Weissman AM. Mdm2 is a RING finger-dependent ubiquitin protein ligase for itself and p53. J Biol Chem. 2000; 275:8945-8951.

30. Xu S, Abbasian M, Patel P, Jensen-Pergakes K, Lombardo CR, Cathers BE, Xie W, Mercurio F, Pagano M, Giegel $\mathrm{D}$ and Cox S. Substrate recognition and ubiquitination of SCFSkp2/Cks1 ubiquitin-protein isopeptide ligase. J Biol Chem. 2007; 282:15462-15470.

31. Rodier G, Coulombe P, Tanguay PL, Boutonnet C and Meloche S. Phosphorylation of Skp2 regulated by CDK2 and $\mathrm{Cdc} 14 \mathrm{~B}$ protects it from degradation by $\mathrm{APC}(\mathrm{Cdh} 1)$ in G1 phase. EMBO J. 2008; 27:679-691.

32. Lin HK, Wang G, Chen Z, Teruya-Feldstein J, Liu Y, Chan $\mathrm{CH}$, Yang WL, Erdjument-Bromage H, Nakayama KI, Nimer S, Tempst P and Pandolfi PP. Phosphorylationdependent regulation of cytosolic localization and oncogenic function of Skp2 by Akt/PKB. Nat Cell Biol. 2009; 11:420-432.

33. Yang CS, Xin HW, Kelley JB, Spencer A, Brautigan DL and Paschal BM. Ligand binding to the androgen receptor induces conformational changes that regulate phosphatase interactions. Mol Cell Biol. 2007; 27:3390-3404. 